\title{
3 Research Square

\section{Heart Rate Variability Predicts Treatment Efficacy in Patients with Chronic Migraine: An Exploratory Study}

Chun-Hsiang Chuang ( $\nabla$ ch.chuang@mx.nthu.edu.tw)

National Tsing Hua University https://orcid.org/0000-0002-5043-8380

Kuan-Lin Lai

Taipei Veterans General Hospital

Jhe-Yu Li

National Taiwan Ocean University

Jung-Tai King

National Yang Ming Chiao Tung University

Wei-Ta Chen

Taipei Veterans General Hospital

Shih-Pin Chen

Taipei Veterans General Hospital

Yen-Feng Wang

Taipei Veterans General Hospital

Hung-Yu Liu

Taipei Veterans General Hospital

Fu-Jung Hsiao

National Yang Ming Chiao Tung University

Li-Ling Hope Pan

National Yang Ming Chiao Tung University

Shuu-Jiun Wang

Taipei Veterans General Hospital https://orcid.org/0000-0001-5179-5358

\section{Research Article}

Keywords: chronic migraine, heart rate variability, electrocardiogram, monthly headache days, flunarizine, clustering

Posted Date: August 23rd, 2021

DOI: https://doi.org/10.21203/rs.3.rs-841353/v1 
License: (c) (i) This work is licensed under a Creative Commons Attribution 4.0 International License. Read Full License 


\title{
Heart Rate Variability Predicts Treatment Efficacy in Patients with Chronic Migraine: An Exploratory Study
}

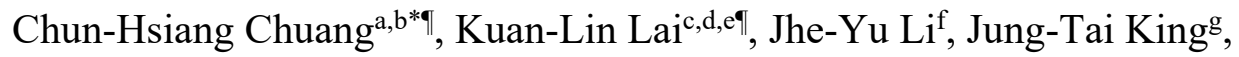
Wei-Ta Chen ${ }^{\mathrm{c}, \mathrm{d}, \mathrm{e}}$, Shih-Pin Chen ${ }^{\mathrm{c}, \mathrm{d}, \mathrm{e}}$, Yen-Feng Wang ${ }^{\mathrm{c}, \mathrm{d}, \mathrm{e}}$, Hung-Yu Liu ${ }^{\mathrm{c}, \mathrm{d}, \mathrm{e}}$, Fu-Jung Hsiao ${ }^{\mathrm{e}}$, Li-Ling Hope Pan ${ }^{\mathrm{e}}$, Shuu-Jiun Wang,
\end{abstract}

a Center for Education and Mind Sciences, College of Education, National Tsing Hua University, Hsinchu, Taiwan

${ }^{\mathrm{b}}$ Institute of Information Systems and Applications, College of Electrical Engineering and Computer Science, National Tsing Hua University

${ }^{\mathrm{c}}$ Neurological Institute, Taipei Veterans General Hospital, Taipei, Taiwan

${ }^{\mathrm{d}}$ College of Medicine, National Yang Ming Chiao Tung University, Taipei, Taiwan

${ }^{\text {e } B r a i n ~ R e s e a r c h ~ C e n t e r, ~ N a t i o n a l ~ Y a n g ~ M i n g ~ C h i a o ~ T u n g ~ U n i v e r s i t y, ~ T a i p e i, ~ T a i w a n ~}$

${ }_{\mathrm{f}}^{\mathrm{f}}$ Department of Computer Science and Engineering, National Taiwan Ocean University, Keelung, Taiwan

${ }^{\mathrm{g}}$ Institute of Neuroscience, National Yang Ming Chiao Tung University, Taipei, Taiwan

I These authors contributed equally.

* Correspondence should be addressed to either of the following:

Chun-Hsiang Chuang

Center for Education and Mind Sciences, College of Education

Institute of Information Systems and Applications, College of Electrical Engineering and

Computer Science

National Tsing Hua University, Taiwan

No. 521, Nanda Road, Hsinchu, Taiwan, 300193.

Email: ch.chuang@mx.nthu.edu.tw

Shuu-Jiun Wang, MD,

Department of Neurology, Neurological Institute, Taipei Veterans General Hospital, No. 201, Sec. 2, Shi-Pai Road, Bei-Tou District, Taipei, Taiwan, 11217.

Email: sjwang@vghtpe.gov.tw 


\begin{abstract}
Background: Chronic migraine (CM) is a disabling headache disorder. Autonomic nervous system (ANS) disturbance, as evidenced from heart rate variability (HRV) studies, has been documented in patients with episodic migraine and other pain disorders but not specifically in patients with CM. This study aimed to explore whether the HRV in patients with CM was impaired and whether it could be used to predict treatment outcomes.
\end{abstract}

Methods: Patients with CM were recruited, along with age- and gender-matched healthy controls (CTLs). The time-domain, frequency-domain, and nonlinear metrics of HRV were calculated to evaluate the sympathetic and parasympathetic aspects of ANS function in patients with CM before and after 3-month (12 weeks) treatment with flunarizine as well as in CTLs. The patients were asked to keep a headache diary throughout the study period to determine the treatment efficacy.

Results: A total of 81 patients with CM and 58 CTLs completed the study. Most HRV values in patients with CM were significantly lower than those in CTLs, suggesting decreased overall autonomic modulation and parasympathetic hypofunction but not sympathetic dysfunction. By unsupervised clustering analysis, patients with $\mathrm{CM}$ were divided into two cluster groups with high and low HRV at baseline. Patients with high baseline HRV, which was comparable to that of CTLs, showed significantly higher absolute and relative reductions in averaged monthly headache days across a 3-month preventive treatment compared with patients with low baseline HRV (-9.1 days vs. -6.4 days or $-43.2 \%$ vs. $-30.1 \%$, both $p<0.05)$. The HRV increased after preventive treatment in the low-HRV group but not in the highHRV group. 
Conclusions: HRV could distinguish patients with CM from CTLs, indicating the involvement of ANS dysfunction. Moreover, patients with CM having a near-normal baseline HRV, indicating a preserved parasympathetic function and overall ANS modulation reservoir, predicted a better outcome to preventive treatment with flunarizine than those with low HRV.

Trial registration: Neurologic Signatures of Chronic Pain Disorders, NCT02747940. Registered 22 April 2016, https://clinicaltrials.gov/ct2/show/NCT02747940

Keywords: chronic migraine, heart rate variability, electrocardiogram, monthly headache days, flunarizine, clustering 


\section{Introduction}

Patients with migraine experience headaches and other constitutional symptoms such as nausea, vomiting, fatigue, loss of appetite, and palpitation. Owing to these associated autonomic symptoms during or even between migraine attacks, dysfunction of the autonomic nervous system (ANS) has long been regarded as a contributor to the pathogenesis of migraine. Previous studies using various methodologies, including heart rate variability (HRV), demonstrated sympathetic hyperactivity [1-4] and parasympathetic hypoactivity [15] in patients with episodic migraine (EM). Similar findings in other pain-related disorders such as fibromyalgia [6], irritable bowel syndrome [7], neck/shoulder pain [8], and neuropathic pain [9] further strengthen the involvement of ANS dysfunction across different painful conditions [10-12]. Chronic migraine (CM), a subtype of migraine, is an even more disabling headache disorder and is defined as headaches on more than 15 days per month for $\geq 3$ months, with at least 8 of them with migraine in a month [13]. Although CM affects approximately $2 \%$ of the global population $[14,15]$, no study specifically evaluated ANS dysfunction using HRV in patients with CM.

HRV is widely accepted as a noninvasive metric of ANS activity by depicting the fluctuations among successive R-peaks in the electrocardiographic (ECG) signal. Three types of analytics, namely time-domain, frequency-domain, and nonlinear analyses, are commonly used to interpret the HRV, including simultaneous interpretation of sympathetic and parasympathetic functions. As potential biomarkers of painful disorders, HRV metrics have also been used as outcome predictors or surrogate markers of clinical improvement. Higher parasympathetic function at baseline has been shown to be associated with a more favorable clinical prognosis and can predict changes in pain symptoms following psychotherapy in patients with pain-predominant multi-somatoform disorder [16]. Two other studies that involved the application of yoga as a therapeutic intervention in patients with EM disclosed 
that effective treatment causes improvement in the parasympathetic profile and regression in a sympathetic domain $[17,18]$.

In this prospective study, we explored whether patients with CM also harbored ANS dysfunction characterized by parasympathetic hypoactivity and/or sympathetic hyperactivity and whether such dysfunction could predict the clinical outcomes of pharmacological therapy or correlate with clinical improvement. Notably, none of the patients with CM were comorbid with other major systemic or psychiatric disorders, which were associated with ANS dysfunction. The selection of preventive medication, flunarizine, a common migraine preventive agent used in Asia and Europe, is based on one of our prior study which demonstrated its comparable effects, yet with less adverse effects, to topiramate in patients with CM [19]. In addition, flunarizine is not associated with heart rate changes as in other migraine preventive agents. 


\section{Materials and Methods}

\subsection{Ethics statement}

The study protocol (Fig. 1A) was approved by the Institutional Review Board (IRB) of Taipei Veterans General Hospital, Taiwan (VGHTPE-IRB: 2015-10-001BC; ClinicalTrials.gov Identifier: NCT02747940). All participants provided their informed consent for inclusion before they participated in the study.

\subsection{Study design}

Patients were recruited from the Headache Clinic of Taipei Veterans General Hospital and evaluated by neurologists. The study consisted of a prospective baseline period of 4 weeks (Week -4 to Week 0 ) to record the baseline clinical profile and a treatment period lasting 12 weeks after enrollment (Weeks 0-12, Fig. 1A). At the first visit, all potential participants completed a structured headache questionnaire for collecting information on their headache profile and for assessing disease severity (Migraine Disability Assessment), psychological disturbance (Beck Depression Inventory [BDI] and Hospital Anxiety and Depression Scale), and stress level (Perceived Stress Scale). Eligible patients who met the ICHD-3 $\beta$ criteria for CM [20] were asked to maintain headache diaries since the prospective baseline period. Subjects with $\geq 14$ headache days and $\geq 8$ migraine days within these 4 weeks who also met the inclusion criteria (see below) were then started on flunarizine as a preventive treatment that lasted for 12 weeks. The initial dosage of flunarizine was $5 \mathrm{mg} / \mathrm{day}$, which could be titrated up to $10 \mathrm{mg} /$ day as per the patients' tolerance. Two ECG examinations were performed for each patient: one during the baseline period and the other after the completion of 12-week treatment (Fig. 1A). The detailed methods are described below. 


\subsection{Subjects}

Inclusion criteria

Subjects aged 20-70 years who met the ICHD-3 $\beta$ criteria for $\mathrm{CM}$ at the first visit were required to fulfill the following additional criteria to be enrolled in this study. Eligible subjects experienced $\geq 14$ days of headache lasting 2 hours during the baseline period. On $\geq 8$ of these days, subjects were required to have experienced migrainous headache, defined as moderate to severe headache with one or more of the following features: unilateral pain or pain that is worse on one side of the head, pulsatile nature, photophobia, and phonophobia, nausea or vomiting, or pain that worsens by routine physical activity or is responsive to acute migraine treatment [19]. To avoid the interference or lasting effects of other preventive medications, this study recruited prophylaxis-naïve patients who did not receive any preventive medications (such as beta-blockers, antidepressants, antiepileptic drugs, or calcium channel blockers), botulinum toxin injection, or acupuncture therapy before participating in this study.

\section{Exclusion criteria}

Patients with any of the following conditions were excluded from the study: [1] headache type other than $\mathrm{CM}$, [2] migraine onset after the age of 50 years, [3] CM onset after the age of 60 years, [4] pregnancy or nursing status, [5] history of the hepatic or renal disorder or other severe systemic diseases, [6] psychiatric diagnosis of major psychiatric disorders or severe depression (BDI score $\geq 30$ at Visit 1), and [7] use of medications that may interfere with autonomic function (e.g., drugs with antiarrhythmic properties). 
Enrollment of healthy controls

Age- and gender-matched healthy controls (CTLs) were recruited from a nearby neighborhood through an advertisement approved by the IRB. The CTLs were not enrolled if they had the following conditions: [1] headache disorder other than infrequent tension-type headache (less than once per month), [2] pregnancy or nursing status, [3] systemic diseases or major psychiatric disorders, and [4] use of medications that may interfere with autonomic functions.

\subsection{Outcome measurement}

Throughout the study period, patients were instructed to maintain a headache diary, which was used to calculate monthly headache days (MHDs) as the outcome measure. The primary end points were the mean change in the MHD reduction as well as the percentage change between the baseline period and the 12-week period during prevention [21].

\subsection{ECG recording and heart rate variability analysis}

As shown in Fig. 1B, a single-lead electrode pair with one electrode placed under the right clavicle at the mid-clavicular line and the other placed on the left upper abdomen was used to record resting-state ECG through the NuAmps system (Compumedics Limited, Australia) in the bipolar lead II configuration (negative and positive, RA-LL). The sampling frequency was $500 \mathrm{~Hz}$. Subjects were informed not to drink coffee within 24 hours before each ECG recording.

Figure 2 summarizes the flowchart of the signal processing, standard short-term ( $5 \mathrm{~min})$ HRV analysis [21] and the statistical analysis in this study. First, the R-wave peak of the QRS complexes in the ECG was detected using the Pan-Tompkins algorithm [22]. RR intervals, which denoted the time elapsed between two successive R-waves of the QRS complexes, 
were then calculated for further HRV analysis. Second, by using Kubios HRV Premium (version 3.3.0, released in June 2019)[23], each RR interval time series was trimmed to a 5min epoch and processed with an automatic artifact correction algorithm to detect and correct ectopic, missed, and extra beats [24]. Third, HRV metrics were assessed through timedomain, frequency-domain, and nonlinear analyses. In this study, a total of eight HRV metrics were adopted. The time-domain metrics included standard deviation of normal-tonormal RR interval (SDNN), root mean square of successive RR interval difference (RMSSD), and triangular interpolation of normal-to-normal interval (TINN). The frequencydomain metrics were decomposed by fast Fourier transform, resulting in absolute powers of the very-low-frequency band (VLF $<0.04 \mathrm{~Hz}$ ), the low-frequency band ( $\mathrm{LF}=0.04-0.14 \mathrm{~Hz}$ ), and the high-frequency band $(\mathrm{HF}=0.14-0.4 \mathrm{~Hz})$. The nonlinear HRV metrics derived by analyzing the geometry of consecutive RR intervals on the Poincaré plot [25] were SD1 and SD2. In general, HF power, RMSSD, and SD1 are regarded as indicators of parasympathetic activity, LF power as indicators of sympathetic activity, while SDNN, TINN, SD2 represent the overall modulation reservoir of the ANS [26].

\subsection{Baseline HRV analysis and correlation with treatment outcome}

Two hypotheses were tested in this study.

As mentioned in the introduction, previous studies showed ANS dysfunction in patients with EM and various chronic pain disorders. Therefore, this study hypothesized that ANS dysfunction might also occur in patients with CM. Specifically, this study compared baseline (pre-treatment) HRV metrics, including SDNN (in ms), RMSSD (in ms), TINN (in ms), VLF

power (in $\mathrm{ms}^{2}$ ), LF power (in $\mathrm{ms}^{2}$ ), HF power (in $\mathrm{ms}^{2}$ ), SD1 (in ms), and SD2 (in ms), in patients with CM with those of the CTLs by using independent sample $t$-tests. The HRV 
metrics that could distinguish CM from CTL would also be addressed to identify abnormalities in parasympathetic and sympathetic activities.

Additionally, this study hypothesized that differences in ANS function among patients might reveal the differences in their treatment outcomes. To test this hypothesis, patients with CM were divided into two groups based on their baseline HRV using the k-means clustering algorithm [27, 28]. Before subgrouping patients, the number of metrics was reduced by calculating the similarity between HRV values using Ward's method [27]. This agglomerative hierarchical clustering algorithm constructed a dendrogram that provided similar information about merging metrics into branches. By selecting a single representative metric from each branch, the number of metrics was reduced. The HRV values of selected metrics were then scaled with the z-score normalization in k-means clustering. The difference in treatment outcomes between subgroups congregated by baseline HRVs was compared using independent $t$-tests. ANCOVA replaced the $t$-test if any covariance existed.

This study further tested whether the differences in each selected HRV parameter before and after treatment is correlated with the extent of clinical improvement for evaluating whether these parameters could serve as surrogate biomarkers.

Statistical analyses were performed using SPSS ver. 22.0 (IBM, Armonk, NY, USA). All multiple comparisons were conducted by adjusting $p$ values using the false discovery rate (FDR) method [29]. The significance level was set as 0.05 . Figures were created using MATLAB R2020a. 


\section{Results}

\subsection{Demographics}

As shown in Fig. 1C, $135 \mathrm{CM}$ and 70 CTL were screened. In total, 81 patients with CM (female ratio $=92.6 \%$, mean age $38.1 \pm 11.4$ years) and 58 age- and gender-matched CTLs (female ratio $=84.5 \%$, mean age $40.6 \pm 9.2$ years) completed the study after excluding ineligible conditions, and were used for final analyses. The demographics and clinical characteristics of the study participants are shown in Table 1. The MHDs recorded by the CM group at baseline $\left(\mathrm{MHD}_{0}\right)$ were $21.6 \pm 6.0$ days. After treatment, the MHDs of the first (weeks 1-4), second (weeks 5-8), and third (weeks 9-12) months were 16.7 $\pm 8.3,13.5 \pm 8.1$, and $12.1 \pm 8.5$, respectively. The changes in MHDs demonstrated a significantly decreasing trend (repeated measures ANOVA with Greenhouse-Geisser correction, $p<0.001$ ), that is, $\mathrm{MHD}_{3}<\mathrm{MHD}_{2}<\mathrm{MHD}_{1}<\mathrm{MHD}_{0}$.

\subsection{Comparison of 5-min HRV between CTLs and patients with CM at baseline}

Most HRV metrics at baseline showed statistically significant differences between the CM and CTL groups (Fig. 3A) (independent sample $t$-test, FDR-adjusted $p<0.05$ ), except for LF. The SDNN (Fig. 3B), RMSSD, TINN, VLF, HF (Fig. 3C), SD1, and SD2 (Fig. 3D) of patients with CM were all significantly lower than those of CTLs. The detailed data of baseline HRVs are summarized in Table 1.

\subsection{Similarity between HRV metrics}

Fig. 4A shows the dendrogram of hierarchical clustering analysis on eight selected HRV metrics collected from the CM group. In the dendrogram, two clusters with the smallest 
dissimilarity (i.e., linkage) were merged into a new cluster. The results showed that the RMSSD merged with SD1 at the linkage of almost zero and then with HF at the linkage of 15.06. The SDNN merged with SD2 at the linkage of 1.14, with TINN at the linkage of 4.79, and then with LF at the linkage of 46.80. Subsequently, all the metrics mentioned above combined together at the linkage of 106.83 and then merged with the remaining metric, VLF, at the linkage of 192.22. To group these metrics into distinct HRV branches, one of the possible cut-off points of the dendrogram was set to locate at around 50 to 100 , resulting in three main branches. The first branch consisted of RMSSD, SD1, and HF; the second branch consisted of SDNN, SD2, TINN, and LF; and the third branch consisted of only VLF power.

For patient clustering, one of the metrics in each branch was selected to reduce the data dimensionality to three before the $k$-mean clustering. As each branch contained at least one frequency-domain metric, the metrics initially selected to be used for further analysis were HF, LF, and VLF. However, in the second branch, SDNN replaced LF because the latter failed to differentiate the CM from CTL groups (Fig. 3A).

\subsection{Difference in treatment efficacy between patient clusters}

As shown in the 3D scatter plot in Fig. 4B, 81 patients were assigned to two disjoint clusters. Cluster 1 (blue) consisted of 49 patients (46 female) aged $42.0 \pm 9.7$ years, and Cluster 2 (red) consisted of 32 patients (29 female) aged 32.2 \pm 11.4 years. Age exhibited a statistically significant difference between the two clusters $(p<0.001)$ but gender did not (Table 2).

As summarized in Table 2, all eight selected HRVs in Cluster 2 were significantly larger than those in Cluster 1 (FDR-adjusted $p<0.001$, ANCOVA with age as the covariate). 
After consecutive treatment for three months, the mean absolute and relative MHD reduced by 9.1 days and 6.4 days or $43.2 \%$ and $30.1 \%$ in the high- and low-HRV clusters, respectively (rmANCOVA with age as the covariate, both $p<0.05$ ), albeit their baseline MHDs did not differ. Table 2 presents the detailed information, including between-group comparison regarding mean MHD reduction after 4-week, and 8-weeks, as explorative analysis.

\subsection{Difference in HRV metrics after receiving treatment}

Figure 5 shows the HRV before and after the 3-month treatment. For the low-HRV group, all HRV metrics, except for the HF, significantly increased after treatment $(p<0.05)$. However, their baseline and after-treatment HRVs were still significantly lower than those of CTLs $(p<0.05)$. By contrast, most of the HRV of the high-HRV group were comparable to those of the CTL group $(p>0.05)$ either before or after treatment. The VLF is the only HRV parameter that significantly increased after treatment in the high-HRV group.

\section{Discussion}

The main findings of this study are summarized below. First, the MHDs of patients reduced as treatment continued. Second, the HRV metrics, SDNN, RMSSD, TINN, VLF, HF, SD1, and SD2, of patients with CM were significantly lower than those of CTLs. Third, HRV metrics could be divided into three categories. Fourth, patients with higher pretreatment baseline HRVs exhibited a greater reduction of MHDs than those with lower HRVs. Taken together, the results indicate that baseline HRV could predict treatment outcomes in patients with CM. 


\subsection{Autonomic impairment in patients with chronic migraine}

This study showed that the HRV metrics, except for LF, of patients with CM were significantly lower than those of healthy individuals, implying a generalized state of autonomic disturbance in patients with CM. Although studies on HRV analysis in CM are lacking, a considerable number of studies showed that different headache disorders are associated with ANS dysregulation [30], including EM [4], cluster headache [31], tensiontype headache [32], and trigeminal autonomic cephalalgia [33]. In addition, reduced HRVs are associated with other chronic pain disorders, such as fibromyalgia [10, 34], chronic low back pain [35], chronic neuropathic pain [36] and even in children with chronic pain [37]. The results of this study further demonstrated that patients with $\mathrm{CM}$ had decreased parasympathetic modulation [10], as revealed by low HF, SD1, and RMSSD values, and overall reduced autonomic modulation capability, as revealed by low SD2 [38] SDNN [21], and TINN.

LF, a parameter widely used as an indicator of sympathetic activity, did not show between-group differences in this study. A number of studies suggested that LF may not be a measure of sympathetic activity [39-41]. Some studies considered LF as a measure of baroreflex function [39]. Baroreflex dysfunction was found in patients with tensiontype/exertional headache [42], and baroreflex sensitivity increased in patients with migraine [43]. However, studies on the role of LF in the baroreflex function still showed inconsistencies [44]. Regardless of the manner in which LF functioned, LF was the only one out of eight HRV metrics that showed no significant difference between CTLs and patients with CM. This finding is consistent with the conclusion of a recent HRV meta-analysis that no significant difference in LF exists between CTLs and patients with chronic pain [10].

A previous study [45] showed that the difference in VLF between patients with EM and healthy CTLs was not significant. However, this study found that CM was associated with a 
significantly reduced VLF response. This result suggests that frequent episodes of headache could lead to a reduced VLF.

\subsection{Similarity between HRV parameters of patients with CM}

Some of the several metrics used to assess HRVs share similar characteristics. Their relationships have been described using correlation [46], clustering [47], or factor [48] analysis. It has been widely accepted that RMSSD, SD1, and HF characterize the short-term $\operatorname{HRV}[21,25,26,49,50]$ by assessing the parasympathetic activity. SD2, SDNN, and TINN, which represent long-term variability $[25,51]$, correlate with the combination of sympathetic and parasympathetic activities, i.e., the overall autonomic modulation capacity. The grouping results of HRV metrics using Ward's method presented in patients with CM were consistent with previous findings. Two major divisions of HRV metrics, the short- (RMSSD, SD1, and HF) and long-term (SDNN, SD2, TINN, and LF) variabilities, formed their branches. The VLF standing apart from these two branches demonstrated its different response from the rest of the metrics. Additionally, the sequence of clustering in the dendrogram (Fig. 4A) revealed the degree of similarity between metrics for quantifying the amount of variability. For example, SD1 merged with RMSSD at the linkage of almost zero, showing that they might be identical HRV metrics [49].

Although studies categorized the LF as a long-term HRV metric, a considerable discrepancy still exists between LF and other HRV metrics of the same branch. The results observed in our current patients with CM support the conjecture that LF is the last HRV metric to be merged into the long-term HRV group. LF could form a separate branch if the within-group similarity is increased by setting a lower cut-off value for clustering.

\subsection{Treatment outcome prediction}


Flunarizine, one of the preventive medications recommended for migraine prophylaxis [52], was used in this study. Consistent with previous studies including ours [19, 53-55], the current results revealed that MHD significantly reduced from 21.6 \pm 6.0 to $12.1 \pm 8.5$ (Table 1 ) in the overall population with CM. The extent of clinical improvement was similar to that in previous studies mentioned above. Additionally, patients with $\mathrm{CM}$ with a higher baseline HRV achieved a more considerable reduction in the number of headache days after treatment.

HRV has been recommended for various applications, such as for estimating the risk of cardiovascular diseases, cancer, and chronic obstructive pulmonary disease [56]; predicting treatment response in major depressive disorder [57]; or predicting the outcome of patients with brain injury [58]. The current study demonstrated the potential of predicting the efficacy of preventive treatment for patients with $\mathrm{CM}$ based on their baseline $\mathrm{HRV}$, as $\mathrm{CM}$ with a near-normal HRV (i.e., high-HRV group) could show a significantly higher treatment efficacy (Fig. 4). Interestingly, the high-HRV group showed no interval changes after preventive treatment, whereas the low-HRV group demonstrated partial normalization (Fig. 5). The possible mechanisms warrant further discussion. The interaction between pain perception and HRV may be reciprocally related. Thus, as headache improved even in the low-HRV group, a normalization of HRV may be derived from the reduction of pain perception. By contrast, the high-HRV group may already have a robust ANS function that could not be further improved because of the ceiling effect.

\section{4 Limitations}

Our study has some limitations. First, the headache status of patients during ECG recording was not controlled. As HRV may be affected by the presence or absence of pain [59], the difference between CTL and CM groups could be derived from either the state of pain or the trait of the disease. Nevertheless, as patients had a mean MHD of 21.6 at baseline, 
they were most likely at the true ictal period or between two close attacks (i.e., a broader definition of the ictal period), leading to ECG examination of inter-ictal period hard to be recorded. The results observed in this study could be a combination of both state and trait effects. Second, because we recruited a relatively homogenous group of patients with CM (e.g., prophylactics naïve), our findings may not be generalizable to the other populations with CM. For example, the majority of patients with CM may have multiple comorbidities and have been prescribed multiple preventive medications. Third, although we applied a hypothesis-free data-driven method, i.e., the $k$-means clustering algorithm, to classify the patients into high- and low-HRV subgroups, the number of patients in each subgroup differed. Yet, the results remained similar if we compared the same high-HRV subgroup $(n=32)$ to a newly selected, age-, gender-, and number-matched $(n=32)$ low-HRV subgroup. Finally, this study applied only flunarizine as the preventive regimen. Thus, the application of baseline HRV metrics as outcome predictors to other preventive strategies warrant further studies.

\section{Conclusions}

In this study, we recruited patients with CM and kept track of their clinical improvement as well as their baseline HRV and HRV changes after receiving flunarizine treatment for three months. Our findings suggested that the HRV metrics could differentiate patients with $\mathrm{CM}$ from healthy individuals, indicating the involvement of ANS dysfunction in CM. By leveraging the k-means clustering, this study further differentiated patients with a nearnormal HRV from others and found different outcomes after flunarizine treatment between these two groups. Overall, patients with $\mathrm{CM}$ with a near-normal baseline HRV showed a significantly higher treatment response than those with abnormal HRV did. Our findings suggested that the baseline HRV might be used to predict treatment outcomes in patients with CM. 


\section{List of Abbreviations}

CM: Chronic migraine

CTL: Healthy controls

ECG: Electrocardiography

ANS: Autonomic nervous system

HRV: Heart rate variability

MHD: Monthly headache day

MIDAS: Migraine Disability Assessment

HADS-A: The anxiety subscale of the Hospital Anxiety and Depression Scale

HADS-D: The depression subscale of the Hospital Anxiety and Depression Scale

SDNN: Standard deviation of normal-to-normal RR intervals

RMSSD: Root mean square of successive RR interval differences

TINN: Triangular interpolation of normal-to-normal intervals

VLF: Very-low-frequency

LF: Low-frequency

HF: High-frequency 


\section{Declaration:}

\section{Availability of data and materials}

The datasets analyzed during the current study are available from the corresponding author on reasonable request.

\section{Acknowledgments}

We would like to thank the study participants and the research assistants for actively participating.

\section{Funding}

This work was supported by grants from Ministry of Science and Technology of Taiwan (MOST 110-2636-E-007-018 to CH Chuang, MOST 109-2314-B-075-049 to KL Lai, MOST 106-2321-B-010-009, 108-2321-B-010-014-MY2, 108-2321-B-010-001, 108-2314-B-010023-MY3, 109-2321-B-010-006, 110-2321-B-010-005, and 110-2321-B-A49A-502 to SJ Wang), Taipei Veterans General Hospital (V108C-129 to WT Chen), Ministry of Health and Welfare, Taiwan (MOHW 108-TDU-B-211-133001 and 109-TDU-B-211-114001), and Brain Research Center, National Yang-Ming University from The Featured Areas Research Center Program within the framework of the Higher Education Sprout Project by the Ministry of Education in Taiwan.

\section{Author information}

\section{Affiliations}

Center for Education and Mind Sciences, College of Education \& Institute of Information Systems and Applications, College of Electrical Engineering and Computer Science, National Tsing Hua University, No. 521, Nanda Rd., Hsinchu, Taiwan

Chun-Hsiang Chuang

Neurological Institute, Taipei Veterans General Hospital, No. 201, Sec. 2 Shih-Pai Rd, Taipei, Taiwan

Kuan-Lin Lai, Wei-Ta Chen, Shih-Pin Chen, Yen-Feng Wang, Hung-Yu Liu \& Shuu-Jiun Wang 
School of Medicine, National Yang Ming Chiao Tung University, Taipei, Taiwan

Kuan-Lin Lai, Wei-Ta Chen, Shih-Pin Chen, Yen-Feng Wang, Hung-Yu Liu \& Shuu-Jiun Wang

Brain Research Center, National Yang Ming Chiao Tung University, Taipei, Taiwan Kuan-Lin Lai, Wei-Ta Chen, Shih-Pin Chen, Yen-Feng Wang, Hung-Yu Liu, Fu-Jung Hsiao, Li-Ling Hope Pan, Shuu-Jiun Wang

Department of Computer Science and Engineering, National Taiwan Ocean University, Keelung, Taiwan

Jhe-Yu Li

Institute of Neuroscience, National Yang Ming Chiao Tung University, Taipei, Taiwan Jung-Tai King

\section{Contributions}

$\mathrm{CH}$ Chuang: study design, data acquisition, data analysis, drafting initial manuscript, and manuscript revision; KL Lai: study design, data acquisition, data analysis, and manuscript revision; JY Li: drafting initial manuscript and data analysis; JT King: data acquisition; WT Chen: study design and data acquisition; SP Chen: study design and data acquisition; YF Wang: study design and data acquisition; HY Liu: data acquisition; FJ Hsiao: study design; LLH Pan: data acquisition and data analysis; SJ Wang: study design, data acquisition, and manuscript revision. The authors participated in the discussion, provided the comments, read and approved the final manuscript.

\section{Corresponding authors}

Correspondence to Chun-Hsiang Chuang or Shuu-Jiun Wang.

\section{Ethics declarations:}

\section{Ethics approval and consent to participate}

All participants completed informed consent forms after receiving a complete explanation of the study. The Institutional Review Board of Taipei Veterans General Hospital approved the 
study protocol (VGHTPE-IRB: 2015-10-001BC; ClinicalTrials.gov Identifier: NCT02747940).

\section{Consent for publication}

Not applicable.

\section{Competing interests}

CH Chuang, KL Lai, JY Li, JT King, WT Chen, SP Chen, YF Wang, HY Liu, FJ Hsiao, and LLH Pan have no conflict of interest to declare. SJ Wang has served on the advisory boards of Eli Lilly, Daiichi-Sankyo, Taiwan Pfizer and Taiwan Norvatis. He has received honoraria as a moderator from Allergan, Pfizer, Eli Lilly, Bayer, and Eisai. 


\section{Table}

Table 1. Demographic and clinical characteristics of controls and patients with chronic migraine

\begin{tabular}{|c|c|c|c|c|c|c|}
\hline & & $\operatorname{CTL}(n=58)$ & \multicolumn{2}{|c|}{$\mathrm{CM}(n=81)$} & statistics & significance \\
\hline \multicolumn{7}{|c|}{ Demography } \\
\hline \multicolumn{2}{|c|}{ No. of Females } & 49 & \multicolumn{2}{|l|}{75} & $\chi^{2}(1)=2.309$ & none \\
\hline \multicolumn{2}{|c|}{ Age (years) } & $40.6 \pm 9.2$ & \multicolumn{2}{|c|}{$38.1 \pm 11.4$} & $t(137)=1.346$ & none \\
\hline \multicolumn{2}{|c|}{ MIDAS } & - & \multicolumn{2}{|c|}{$35.5 \pm 39.3$} & - & - \\
\hline \multicolumn{2}{|c|}{ BDI } & $4.9 \pm 5.8$ & \multicolumn{2}{|c|}{$11.0 \pm 6.5$} & $t(115)=4.890$ & $* * *$ \\
\hline \multicolumn{2}{|c|}{ HADS-A } & $3.5 \pm 3.3$ & \multicolumn{2}{|c|}{$8.2 \pm 4.0$} & $t(116)=6.362$ & $* * *$ \\
\hline \multicolumn{2}{|c|}{ HADS-D } & $2.8 \pm 2.8$ & \multicolumn{2}{|c|}{$6.0 \pm 3.5$} & $t(116)=4.813$ & $* * *$ \\
\hline \multicolumn{2}{|c|}{ PSS } & $17.9 \pm 8.2$ & \multicolumn{2}{|c|}{$26.3 \pm 8.1$} & $t(116)=5.252$ & $* * *$ \\
\hline \multicolumn{7}{|c|}{ Baseline HRV } \\
\hline \multicolumn{2}{|c|}{ SDNN (ms) } & $38.1 \pm 10.5$ & \multicolumn{2}{|c|}{$25.6 \pm 10.9$} & $t(137)=6.785$ & $* * *$ \\
\hline & (ms) & $30.4 \pm 12.2$ & \multicolumn{2}{|c|}{$22.9 \pm 11.2$} & $t(137)=3.708$ & $* * *$ \\
\hline & & $180.1 \pm 51.1$ & \multicolumn{2}{|c|}{$127.1 \pm 54.2$} & $t(137)=5.825$ & $* * *$ \\
\hline & & $593.2 \pm 419.1$ & \multicolumn{2}{|c|}{$145.0 \pm 230.6$} & $t(81.661)=7.384^{\mathrm{a}}$ & $* * *$ \\
\hline & & $378.6 \pm 356.9$ & \multicolumn{2}{|c|}{$265.5 \pm 357.9$} & $t(137)=1.839$ & none \\
\hline & & $522.5 \pm 441.1$ & \multicolumn{2}{|c|}{$288.0 \pm 268.0$} & $t(86.783)=3.60^{\mathrm{a}}$ & $* * *$ \\
\hline & & $21.5 \pm 8.7$ & \multicolumn{2}{|c|}{$16.2 \pm 8.0$} & $t(137)=3.709$ & $* * *$ \\
\hline & & $48.9 \pm 14.1$ & \multicolumn{2}{|c|}{$31.9 \pm 14.0$} & $t(137)=7.021$ & $* * *$ \\
\hline \multicolumn{7}{|c|}{ Monthly Headache Days } \\
\hline vs. & $\mathrm{MHD}_{0}$ & $\mathrm{MHD}_{1}$ & \multicolumn{4}{|c|}{$\mathrm{MHD}_{2} \quad \mathrm{MHD}_{3}$} \\
\hline $\mathrm{MHD}_{0}$ & $21.6 \pm 6.0$ & - & - & \multicolumn{2}{|l|}{-} & \\
\hline $\mathrm{MHD}_{1}$ & $* * *$ & $16.7 \pm 8.3$ & - & - & \multirow{3}{*}{$\begin{array}{l}F(2.418,193.407) \\
=75.224^{b}\end{array}$} & \multirow{3}{*}{$* * *^{\mathrm{c}}$} \\
\hline $\mathrm{MHD}_{2}$ & $* * *$ & $* * *$ & \multirow{2}{*}{$\begin{array}{l}13.5 \pm 8.1 \\
* *\end{array}$} & - & & \\
\hline $\mathrm{MHD}_{3}$ & $* * *$ & $* * *$ & & $12.1 \pm 8.5$ & & \\
\hline
\end{tabular}

The upper part of the table summarizes the numbers, gender, age, and baseline HRVs of the recruited participants. The diagonal numbers shown on the lower part of the table are the monthly headache days (MHDs) recorded at the baseline $\left(\mathrm{MHD}_{0}\right)$ and after receiving $1\left(\mathrm{MHD}_{1}\right), 2\left(\mathrm{MHD}_{2}\right)$, and $3\left(\mathrm{MHD}_{3}\right)$ months of treatment. The changes in MHD are evaluated by repeated-measures ANOVA, followed by least significant difference pairwise multiple comparison tests. The asterisk indicates significance at $p<0.05(*), p<0.01(* *)$, and $p<$ $0.001(* * *)$.

${ }^{a}$ Welch's test is performed due to unequal variance.

${ }^{\mathrm{b}}$ Critical values of $\mathrm{F}(3,240)=2.642$ is set at the significance level of 0.05 .

${ }^{c} \mathrm{p}$-value is corrected by Greenhouse-Geisser procedure repeated-measures ANOVA. 
Table 2. Demographics and baseline HRV of two CM clusters (low- and high-HRV).

\section{Demography}

No. of Females

Age (years)

Monthly headache days at $21.3 \pm 6.4$

baseline, $\mathrm{MHD}_{0}$ (days)

$42.0 \pm 9.7$

Low-HRV High-HRV

$(n=49) \quad(n=32)$

$46 \quad 29$

Baseline HRV

\begin{tabular}{|c|c|c|c|c|c|c|}
\hline \multirow{3}{*}{\multicolumn{2}{|c|}{ I }} & RMSSD (ms) & $17.1 \pm 7.8$ & $31.9 \pm 9.6$ & $\mathrm{~F}(1,78)=55.611$ & $* * *$ \\
\hline & & $\mathrm{HF}\left(\mathrm{ms}^{2}\right)$ & $150.6 \pm 131.8$ & $498.6 \pm 287.7$ & $\mathrm{~F}(1,78)=46.831$ & $* * *$ \\
\hline & & SD1 (ms) & $12.1 \pm 5.6$ & $22.6 \pm 6.8$ & $\mathrm{~F}(1,78)=55.601$ & $* * *$ \\
\hline \multirow{5}{*}{ 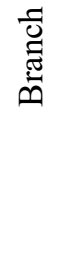 } & \multirow[t]{4}{*}{ II } & SDNN (ms) & $18.3 \pm 5.9$ & $36.7 \pm 6.6$ & $F(1,78)=133.758$ & $* * *$ \\
\hline & & TINN (ms) & $92.9 \pm 28.6$ & $179.5 \pm 40.4$ & $F(1,78)=96.277$ & $* * *$ \\
\hline & & $\mathrm{LF}\left(\mathrm{ms}^{2}\right)$ & $109.6 \pm 83.3$ & $504.3 \pm 471.6$ & $\mathrm{~F}(1,78)=30.462$ & $* * *$ \\
\hline & & $\mathrm{SD} 2(\mathrm{~ms})$ & $22.6 \pm 7.3$ & $46.2 \pm 8.7$ & $\mathrm{~F}(1,78)=134.188$ & $* * *$ \\
\hline & III & $\operatorname{VLF}\left(\mathrm{ms}^{2}\right)$ & $49.2 \pm 46.9$ & $291.6 \pm 305.3$ & $F(1,78)=19.617$ & $* * *$ \\
\hline \multicolumn{7}{|c|}{$\begin{array}{l}\text { Averaged Monthly Headache Days relative to Baseline } \\
\text { difference (in days) }\end{array}$} \\
\hline & & $\Delta \mathrm{MHD}_{1}$ & $-3.9 \pm 5.7$ & $-6.3 \pm 7.1$ & $\mathrm{~F}(1,78)=3.422$ & none \\
\hline & & $\overline{\Delta \mathrm{MHD}}_{2}$ & $-5.3 \pm 5.7$ & $-8.3 \pm 6.3$ & $\mathrm{~F}(1,78)=7.324$ & $*$ \\
\hline & & $\overline{\Delta \mathrm{MHD}}_{3}$ & $-6.4 \pm 5.9$ & $-9.1 \pm 6.2$ & $F(1,78)=6.246$ & $*$ \\
\hline \multicolumn{7}{|c|}{ ratio (in \%) } \\
\hline & & $\overline{\mathrm{rMHD}}_{1}$ & $81.1 \pm 29.3$ & $69.8 \pm 30.6$ & $F(1,78)=2.633$ & none \\
\hline & & $\overline{\mathrm{rMHD}}_{2}$ & $75.6 \pm 28.5$ & $60.9 \pm 26.2$ & $F(1,78)=6.245$ & $*$ \\
\hline & & $\overline{\mathrm{rMHD}}_{3}$ & $69.9 \pm 28.1(-30.1 \%)$ & $56.8 \pm 25.6(-43.2 \%)$ & $F(1,78)=5.150$ & $*$ \\
\hline
\end{tabular}

$\overline{\Delta \mathrm{MHD}}_{k}=\frac{1}{k} \sum_{1}^{k}\left(\mathrm{MHD}_{k}-\mathrm{MHD}_{0}\right)$ and $\overline{\mathrm{rMHD}}_{k}=\frac{1}{k} \sum_{1}^{k} \mathrm{MHD}_{k} / \mathrm{MHD}_{0}$, where $k=0,1,2$, and 3, indicating the baseline, one-, two-, and three-month after treatment, respectively.

The comparison of baseline HVR parameters between groups were conducted by ANCOVA, with age as the covariate. The critical values of $\chi^{2}(1)=[0.001,5.024], t(79)=[-1.991,1.991]$, and $\mathrm{F}(1,78)=3.96$ at the significance level of 0.05 , where the chi-squared test and $t$ test were two-sided. The asterisk indicates significance at $p<0.05(*), p<0.01(* *)$, and $p<0.001(* * *)$. 


\section{Figures}
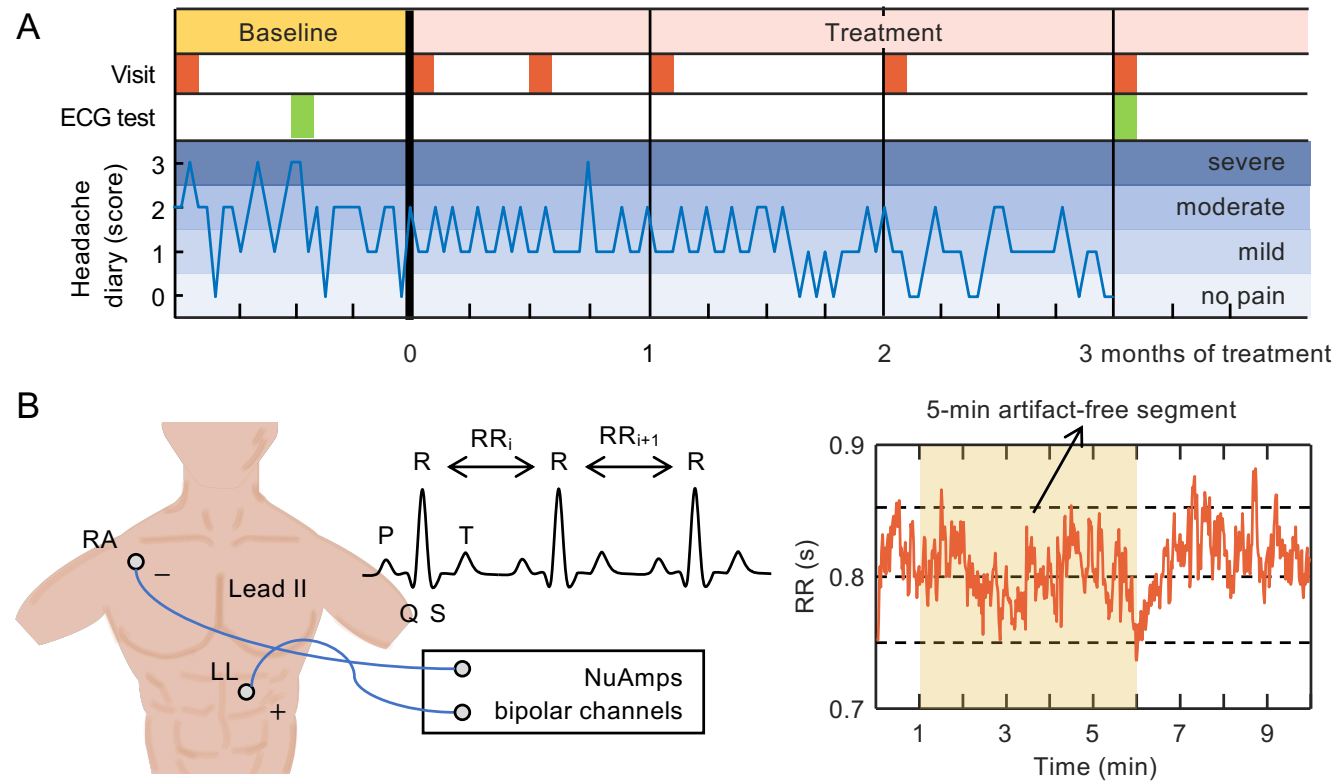

C

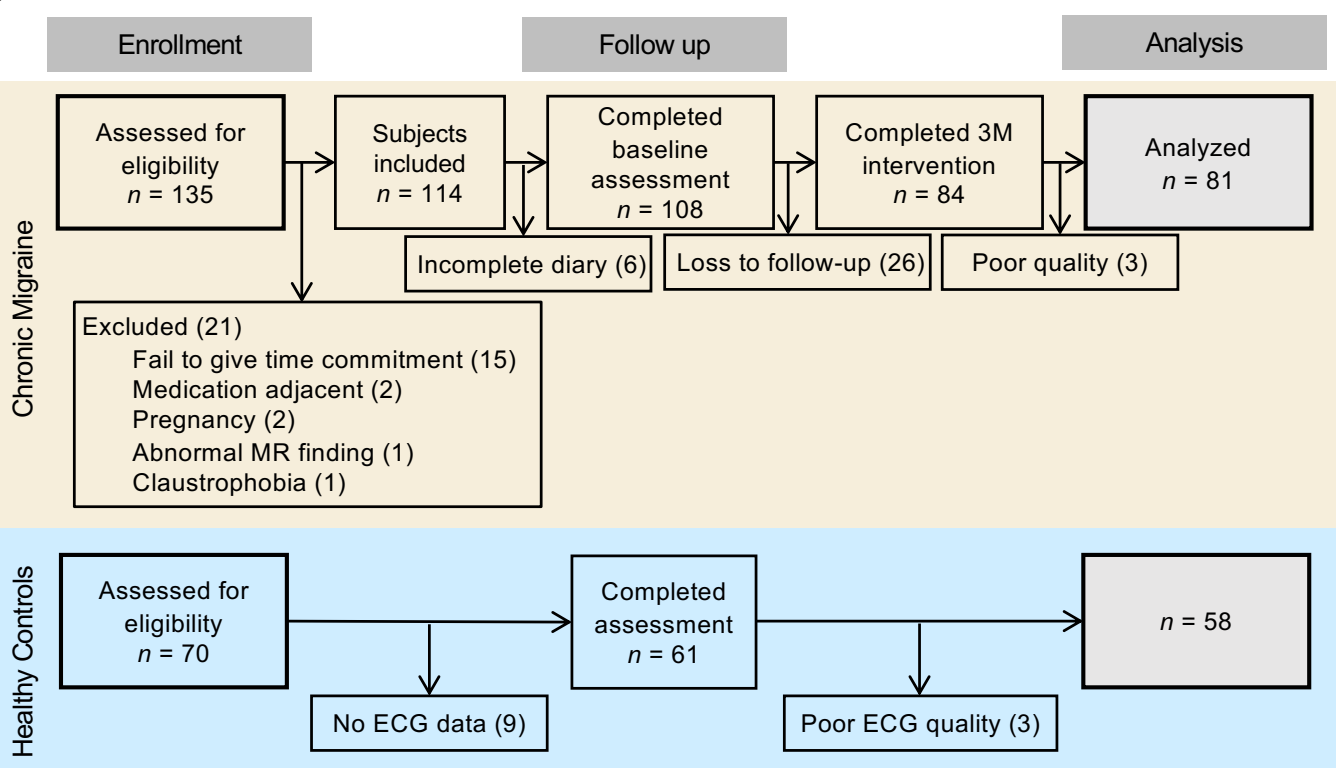

Fig. 1. Experimental design and data collection

(A) Experimental study protocol. In this prospective trial design, the baseline, 4 weeks before the treatment, was followed by a 12 -week treatment. One baseline (pre-treatment) resting-state ECG test was conducted during the first visit. The treatment started after the first follow-up visit. Another (post-treatment) ECG was conducted after the 12-week treatment. Throughout the experiment, each patient was instructed to maintain a headache diary and rate their pain from no pain, mild, moderate, to severe. (B) ECG test. The ECG signal was recorded for 10 minutes through the NuAmps system in the bipolar lead II configuration (negative and positive, RA-LL). The RR, the interval between successive $\mathrm{R}$ peaks in ECG wave, was used for heart rate variability analysis. (C) Recruitment of controls and patients with chronic migraine. 


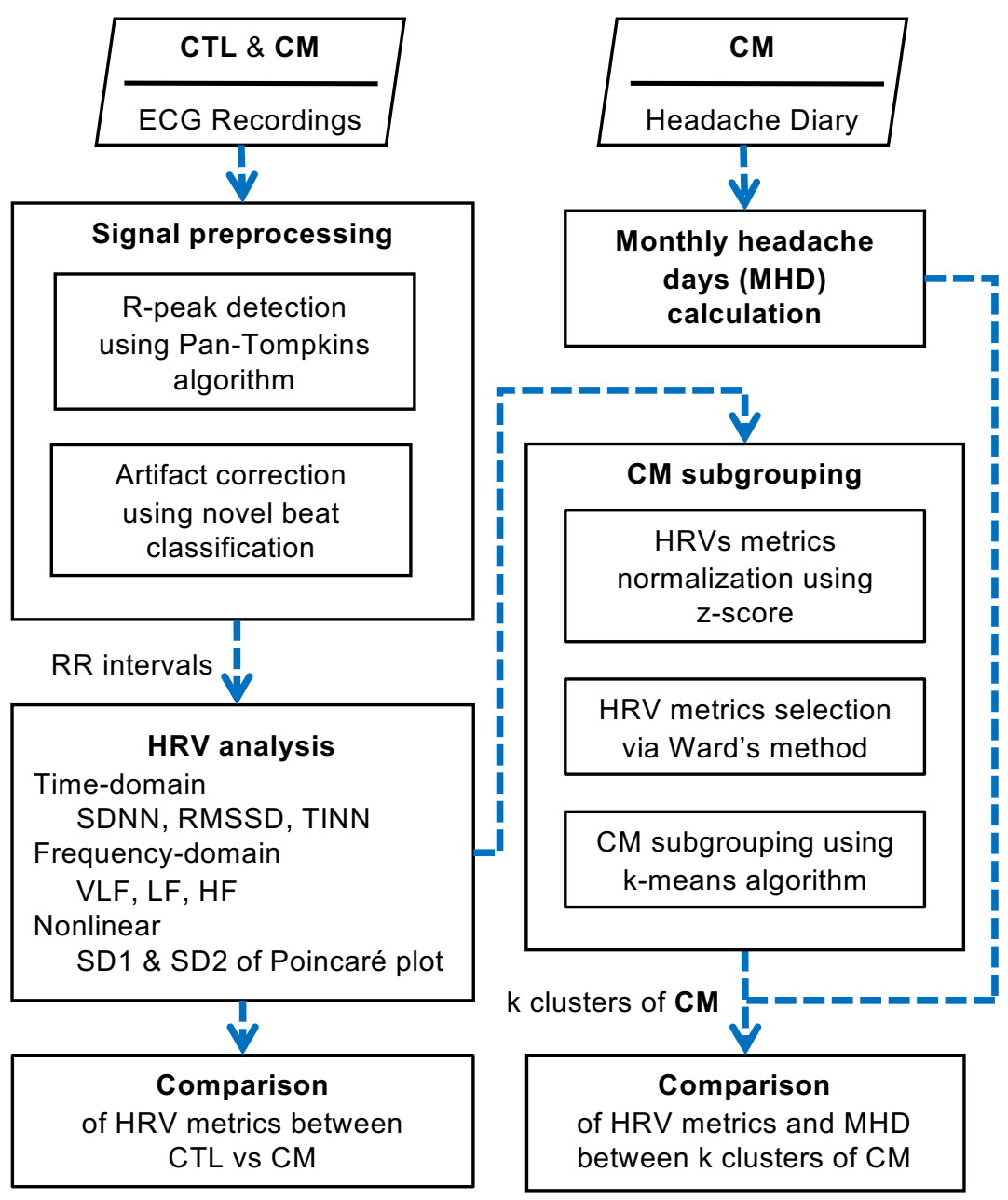

Fig. 2. Flow diagram of signal processing and data analysis

The main steps of this study include signal preprocessing, heart rate variability (HRV) analysis, chronic migraine (CM) subgrouping, and calculation of mean headache days (MHDs). Two main results presented in this study are (1) the comparison of HRV metrics between controls and patients with CM, and (2) the comparison of HRV metrics and MHDs between the subgroups of patients with CM. 


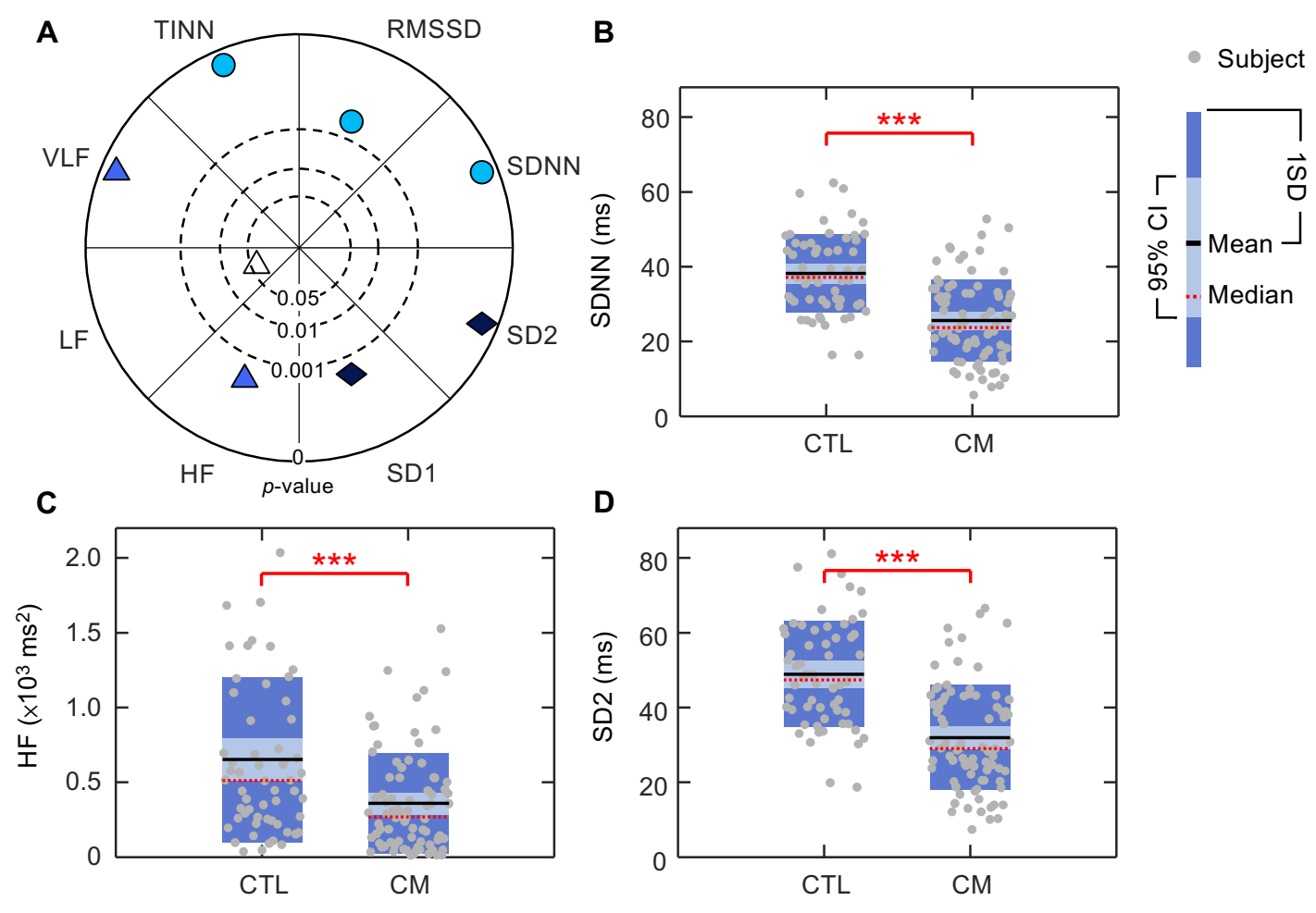

Fig. 3. Comparisons of HRV between controls and patients with chronic migraine (A) The $p$-values of independent $t$ tests comparing HRV, where p-values were corrected for false discovery rate. The $p$-value located in the outer circle is smaller than that located in the inner circle. The $p$-value located in the innermost circle indicates a nonsignificant difference in HRV between the two groups. Three HRV metrics, namely (B) SDNN (ms), (C) HF $\left(\times 10^{3} \mathrm{~ms}^{2}\right)$, and (D) SD2 (ms), of patients with chronic migraine (CM) were compared with those of the controls (CTLs), where three asterisks denote significant differences at $p<0.001$ of independent $t$ tests. Each dot represents either a CTL participant or a patient with CM. Dark and light blue boxes indicate the ranges of plus or minus one standard deviation (SD) and 95\% confidence interval (CI), respectively. 
A

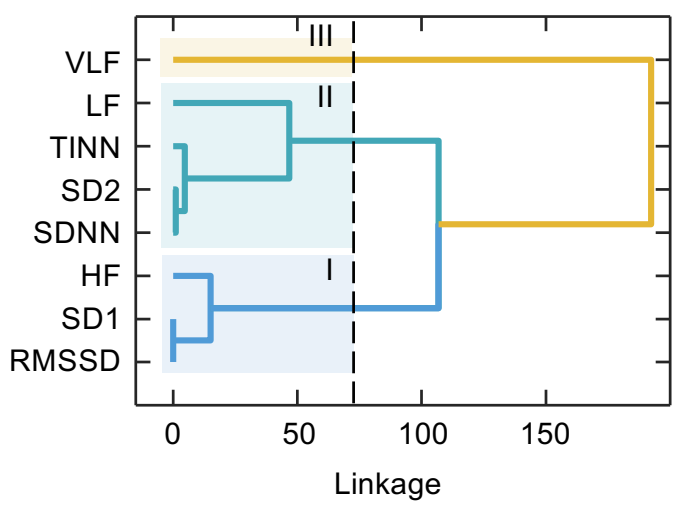

C

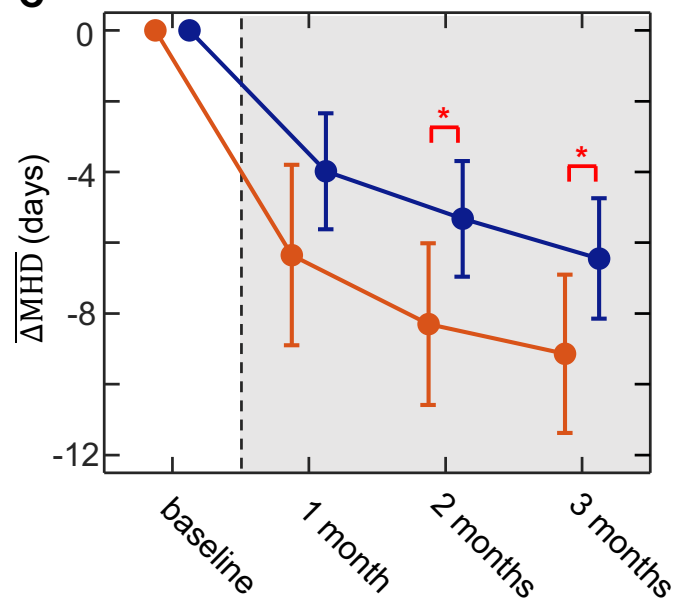

B

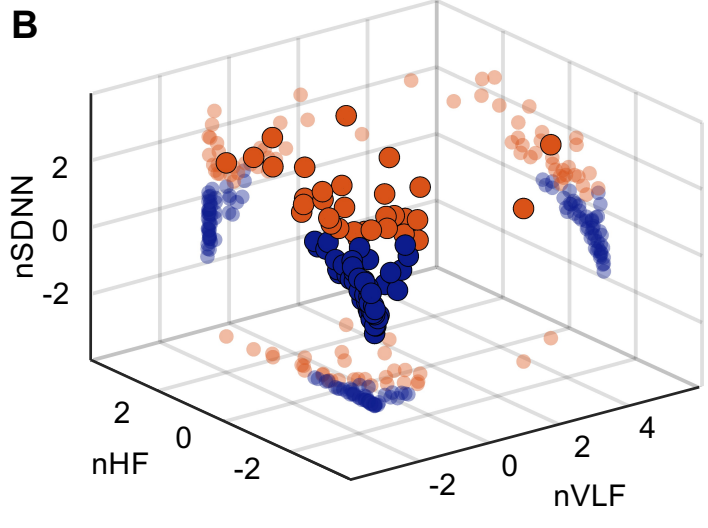

- Low-HRV $\bullet$ High-HRV

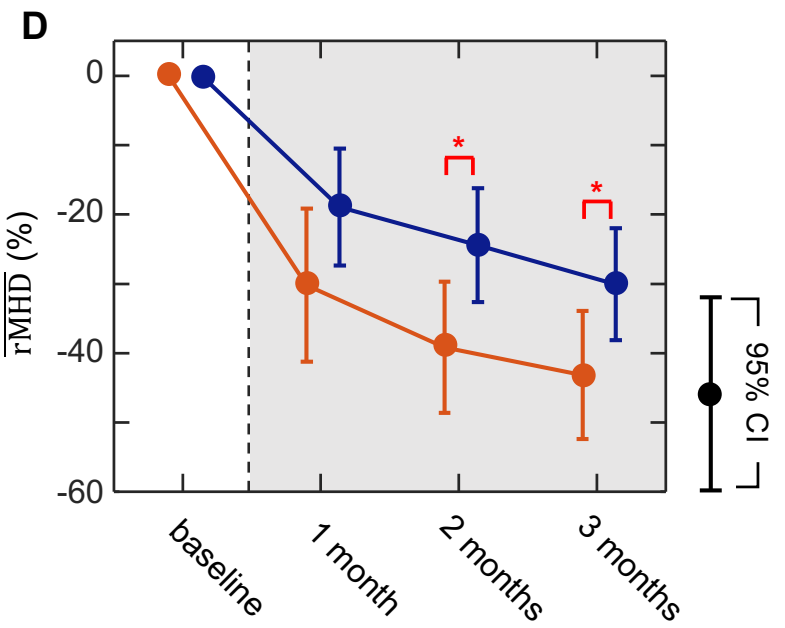

Fig. 4. Chronic migraine subgroups and treatment efficacy

(A) The dendrogram, built by agglomerative hierarchical clustering, reveals the similarity between heart rate variability (HRV) metrics. The dashed line indicates the cut-off point of the clustering, which locates between the linkage of 50 and 100. (B) The 3D scatter plot of groups of patients with chronic migraine (CM) with low and high HRV, where each circle represents the normalized high frequency, very low frequency, and standard deviation of normal-to-normal responses of a patient with CM. (C) The changes in $\overline{\Delta M H D}$. The interaction effect between the MHD and the cluster is statistically significant (rmANCOVA with age as the covariate and Greenhouse-Geisser correction, F(1.577, $122.975)=4.821, p<0.05$ ). (D) The changes in $\overline{r M H D}$. The interaction effect between the MHD and the cluster is statistically significant (rmANCOVA with age as the covariate and Greenhouse-Geisser correction, $\mathrm{F}(1.670,130.243)=3.849, p<0.05)$. Blue and red circles represent the low- and high-HRV groups, respectively. The asterisk indicates a significant difference in $\overline{\mathrm{rMHD}}$ between low- and high-HRV groups $(p<0.05)$. 

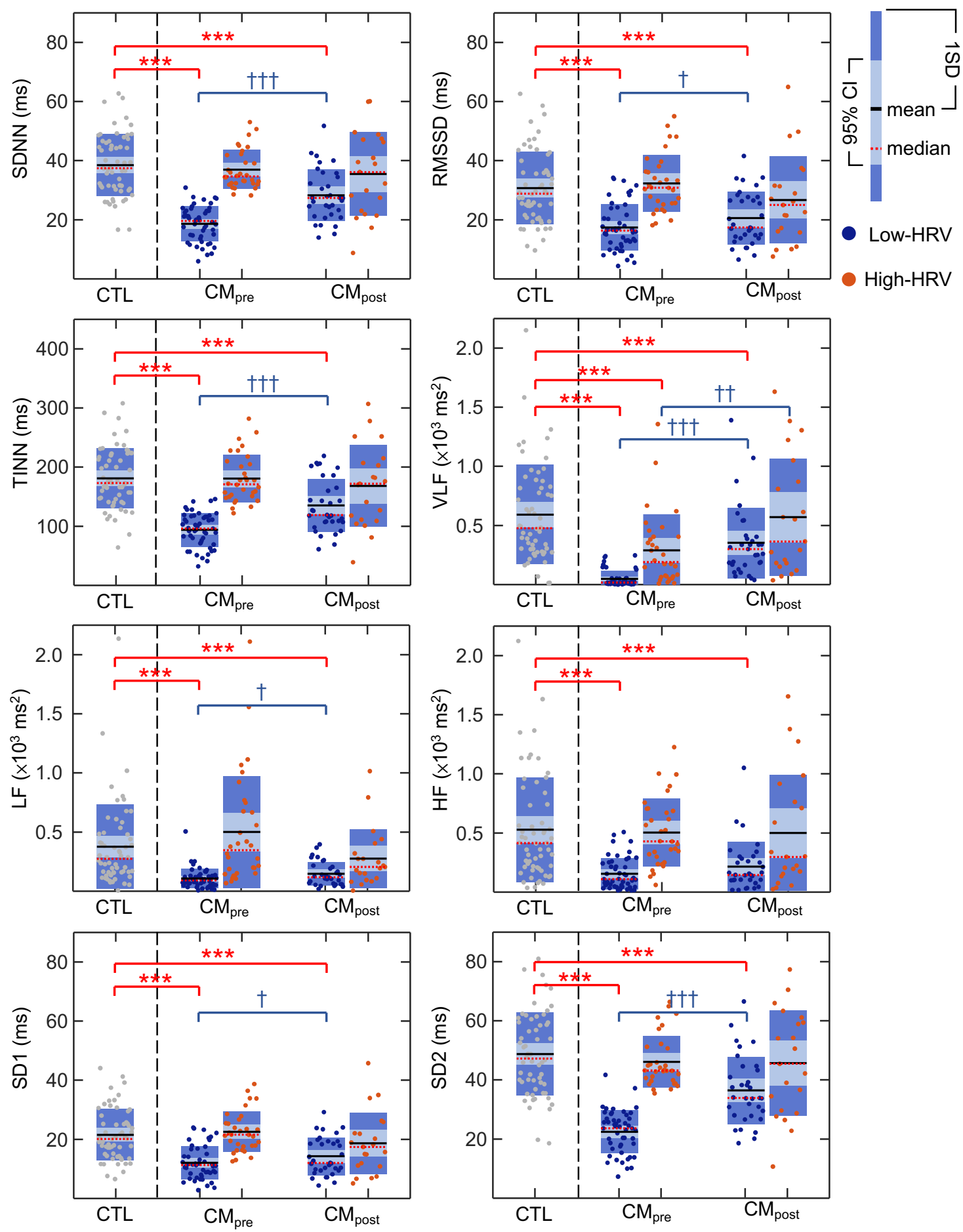

Fig. 5. Comparison of HRV between two patient subgroups with chronic migraine and controls

The HRV of patients with $\mathrm{CM}$ before $\left(\mathrm{CM}_{\text {pre }}\right)$ and after $\left(\mathrm{CM}_{\text {post }}\right)$ the treatment were compared with those of the controls, where the statistical analysis was performed by independent $t$-tests or paired $t$-tests as appropriate and corrected by false discovery rate. The asterisk indicates significance at $p<0.05(*), p<0.01(* *)$, and $p<0.001(* * *)$. 


\section{References}

1. Perciaccante A, Fiorentini A, Valente R, Granata M, Tubani L. Migraine and heart rate variability. Archives of Internal Medicine. 2007;167 20:2264; doi: 10.1001/archinte.167.20.2264-a.

2. Gass JJ, Glaros AG. Autonomic dysregulation in headache patients. Appl Psychophysiol Biofeedback. 2013;38 4:257-63; doi: 10.1007/s10484-013-9231-8.

3. Miglis MG. Migraine and autonomic dysfunction: which is the horse and which is the jockey? Current Pain and Headache Reports. 2018;22 3:19; doi: 10.1007/s11916-018-0671-y.

4. Matei D, Constantinescu V, Corciova C, Ignat B, Matei R, Popescu C. Autonomic impairment in patients with migraine. European Review for Medical and Pharmacological Sciences. 2015;19 20:3922-7.

5. Tabata M, Takeshima T, Burioka N, Nomura T, Ishizaki K, Mori N, et al. Cosinor analysis of heart rate variability in ambulatory migraineurs. Headache. 2000;40 6:457-63; doi: 10.1046/j.1526-4610.2000.00068.x.

6. Raj SR, Brouillard D, Simpson CS, Hopman WM, Abdollah H. Dysautonomia among patients with fibromyalgia: A noninvasive assessment. The Journal of Rheumatology. 2000;27 11:2660-5.

7. Pellissier S, Dantzer C, Canini F, Mathieu N, Bonaz B. Psychological adjustment and autonomic disturbances in inflammatory bowel diseases and irritable bowel syndrome. Psychoneuroendocrinology. 2010;35 5:653-62; doi: 10.1016/j.psyneuen.2009.10.004.

8. Hallman DM, Lindberg LG, Arnetz BB, Lyskov E. Effects of static contraction and cold stimulation on cardiovascular autonomic indices, trapezius blood flow and muscle activity in chronic neck-shoulder pain. European Journal of Applied Physiology 2011;111 8:1725-35; doi: 10.1007/s00421-010-1813-z.

9. Terkelsen AJ, Mølgaard H, Hansen J, Finnerup NB, Krøner K, Jensen TS. Heart rate variability in complex regional pain syndrome during rest and mental and orthostatic stress. Anesthesiology. 2012;116 1:133-46; doi: 10.1097/ALN.0b013e31823bbfb0.

10. Tracy LM, Ioannou L, Baker KS, Gibson SJ, Georgiou-Karistianis N, Giummarra MJ. Meta-analytic evidence for decreased heart rate variability in chronic pain implicating parasympathetic nervous system dysregulation. Pain. 2016;157 1:7-29.

11. Koenig J, Falvay D, Clamor A, Wagner J, Jarczok MN, Ellis RJ, et al. Pneumogastric (vagus) nerve activity indexed by heart rate variability in chronic pain patients vompared to healthy controls: A systematic review and meta-analysis. Pain Physician. 2016;19 1:E55-78.

12. Koenig J, Williams DP, Kemp AH, Thayer JF. Vagally mediated heart rate variability in headache patients--a systematic review and meta-analysis. Cephalalgia. 2016;36 3:265-78; doi: 10.1177/0333102415583989.

13. IHS. Headache Classification Committee of the International Headache Society (IHS) The International Classification of Headache Disorders, 3rd edition. Cephalalgia. 2018;38 1:1-211; doi: 10.1177/0333102417738202.

14. Natoli JL, Manack A, Dean B, Butler Q, Turkel CC, Stovner L, et al. Global prevalence of chronic migraine: a systematic review. Cephalalgia. 2010;30 5:599609; doi: 10.1111/j.1468-2982.2009.01941.x.

15. Stark RJ, Ravishankar K, Siow HC, Lee KS, Pepperle R, Wang SJ. Chronic migraine and chronic daily headache in the Asia-Pacific region: A systematic review. Cephalalgia. 2013;33 4:266-83; doi: 10.1177/0333102412468677. 
16. Angelovski A, Sattel H, Henningsen P, Sack M. Heart rate variability predicts therapy outcome in pain-predominant multisomatoform disorder. Journal of Psychosomatic Research. 2016;83:16-21; doi: 10.1016/j.jpsychores.2016.02.003.

17. Kisan R, Sujan M, Adoor M, Rao R, Nalini A, Kutty BM, et al. Effect of Yoga on migraine: A comprehensive study using clinical profile and cardiac autonomic functions. International Journal of Yoga 2014;7 2:126-32; doi: 10.4103/09736131.133891.

18. Vasudha MS, Manjunath NK, Nagendra HR. Changes in MIDAS, perceived stress, frontalis muscle activity and non-steroidal anti-inflammatory drugs usage in patients with migraine headache without Aura following Ayurveda and Yoga compared to controls: An open labeled non-randomized study. Annals of Neurosciences. 2018;25 4:250-60; doi: 10.1159/000492269.

19. Lai KL, Niddam D, Fuh JL, Chen SP, Wang YF, Chen WT, et al. Flunarizine versus topiramate for chronic migraine prophylaxis: a randomized trial. Acta Neurologica Scandinavica. 2017; 135 4:476-83; doi: 10.1111/ane.12626.

20. IHS. The International Classification of Headache Disorders, 3rd edition (beta version). Cephalalgia. 2013;33 9:629-808; doi: 10.1177/0333102413485658.

21. Shaffer F, Ginsberg JP. An overview of heart rate variability metrics and norms. Frontiers in Public Health. 2017;5 258:1-17; doi: 10.3389/fpubh.2017.00258.

22. Pan J, Tompkins WJ. A Real-Time QRS Detection Algorithm. IEEE Transactions on Biomedical Engineering. 1985;BME-32 3:230-6; doi: 10.1109/TBME.1985.325532.

23. Tarvainen MP, Niskanen JP, Lipponen JA, Ranta-Aho PO, Karjalainen PA. Kubios HRV - Heart rate variability analysis software. Computer Methods and Programs in Biomedicine. 2014;113 1:210-20; doi: 10.1016/j.cmpb.2013.07.024.

24. Lipponen JA, Tarvainen MP. A robust algorithm for heart rate variability time series artefact correction using novel beat classification. Journal of Medical Engineering \& Technology. 2019;43 3:173-81; doi: 10.1080/03091902.2019.1640306.

25. Brennan M, Palaniswami M, Kamen P. Do existing measures of Poincare plot geometry reflect nonlinear features of heart rate variability? IEEE Transactions on Biomedical Engineering. 2001;48 11:1342-7; doi: 10.1109/10.959330.

26. Task Force of the European Society of Cardiology the North American Society of Pacing Electrophysiology. Heart Rate Variability: Standards of measurement, physiological interpretation, and clinical use. Circulation. 1996;93:1043-65.

27. Ward Jr JH. Hierarchical grouping to optimize an objective function. Journal of the American statistical association. 1963;58 301:236-44.

28. Kanungo T, Mount DM, Netanyahu NS, Piatko CD, Silverman R, Wu AY. An efficient k-means clustering algorithm: Analysis and implementation. IEEE Transactions on Pattern Analysis and Machine Intelligence. 2002;24 7:881-92; doi: 10.1109/TPAMI.2002.1017616.

29. Benjamini Y, Hochberg Y. Controlling the false discovery rate: a practical and powerful approach to multiple testing. Journal of the Royal statistical society: series B (Methodological). 1995;57 1:289-300; doi: 10.1111/j.2517-6161.1995.tb02031.x.

30. Koenig J, Loerbroks A, Jarczok MN, Fischer JE, Thayer JF. Chronic pain and heart rate variability in a cross-sectional occupational sample: Evidence for impaired vagal control. The Clinical Journal of Pain. 2016;32 3:218-25; doi: 10.1097/ajp.0000000000000242.

31. Tubani L, Baratta L, Giorgino F, Delfino M, Fiore G, Golluscio V, et al. Heart rate variability in cluster headache. Annali Italiani di Medicina Interna. 2003;18 1:42-6. 
32. Bidikar MP, Jagtap G, Chakor R. Time domain analysis of heart rate variability in tension type headache. International Journal of Health Sciences and Research. 2015;5:161-5.

33. Léonard G, Chalaye P, Goffaux P, Mathieu D, Gaumond I, Marchand S. Altered autonomic nervous system reactivity to pain in trigeminal neuralgia. Canadian Journal of Neurological Sciences. 2015;42 2:125-31; doi: 10.1017/cjn.2015.10.

34. Reyes del Paso GA, de la Coba P. Reduced activity, reactivity and functionality of the sympathetic nervous system in fibromyalgia: An electrodermal study. PLOS ONE. 2020;15 10:e0241154; doi: 10.1371/journal.pone.0241154.

35. Telles S, Sharma SK, Gupta RK, Bhardwaj AK, Balkrishna A. Heart rate variability in chronic low back pain patients randomized to yoga or standard care. BMC Complementary Medicine and Therapies. 2016;16 279:1-7; doi: 10.1186/s12906016-1271-1.

36. Karri J, Zhang L, Li S, Chen Y-T, Stampas A, Li S. Heart rate variability: a novel modality for diagnosing neuropathic pain after spinal cord injury. Front Physiol. 2017;8 495:1-9; doi: 10.3389/fphys.2017.00495.

37. Evans S, Seidman LC, Tsao JC, Lung KC, Zeltzer LK, Naliboff BD. Heart rate variability as a biomarker for autonomic nervous system response differences between children with chronic pain and healthy control children. J Pain Res. 2013;6:449-57; doi: 10.2147/JPR.S43849.

38. Brennan M, Palaniswami M, Kamen P. Poincaré plot interpretation using a physiological model of HRV based on a network of oscillators. American Journal of Physiology-Heart and Circulatory Physiology. 2002;283 5:H1873-H86; doi: 10.1152/ajpheart.00405.2000.

39. Goldstein DS, Bentho O, Park M-Y, Sharabi Y. Low-frequency power of heart rate variability is not a measure of cardiac sympathetic tone but may be a measure of modulation of cardiac autonomic outflows by baroreflexes. Experimental Physiology. 2011;96 12:1255-61; doi: 10.1113/expphysiol.2010.056259.

40. Houle MS, Billman GE. Low-frequency component of the heart rate variability spectrum: a poor marker of sympathetic activity. American Journal of PhysiologyHeart and Circulatory Physiology. 1999;276 1:H215-H23; doi: 10.1152/ajpheart.1999.276.1.H215.

41. Reyes del Paso GA, Langewitz W, Mulder LJ, van Roon A, Duschek S. The utility of low frequency heart rate variability as an index of sympathetic cardiac tone: a review with emphasis on a reanalysis of previous studies. Psychophysiology. 2013;50 5:477-87; doi: 10.1111/psyp.12027.

42. Khurana RK. Headache in patients with baroreflex failure. Headache. 2006;46 7:1207-9; doi: 10.1111/j.1526-4610.2006.00514_3.x.

43. Nilsen KB, Tronvik E, Sand T, Gravdahl GB, Stovner LJ. Increased baroreflex sensitivity and heart rate variability in migraine patients. Acta Neurol Scand. 2009;120 6:418-23; doi: 10.1111/j.1600-0404.2009.01173.x.

44. Martelli D, Silvani A, McAllen RM, May CN, Ramchandra R. The low frequency power of heart rate variability is neither a measure of cardiac sympathetic tone nor of baroreflex sensitivity. American Journal of Physiology-Heart and Circulatory Physiology. 2014;307 7:H1005-H12; doi: 10.1152/ajpheart.00361.2014.

45. Zhang L, Qiu S, Zhao C, Wang P, Yu S. Heart rate variability analysis in episodic migraine: A cross-sectional study. Frontiers in Neurology. 2021;12 308:1-9; doi: 10.3389/fneur.2021.647092.

46. Hoshi R, Pastre C, Vanderlei L, Godoy M. Poincaré plot indexes of heart rate variability: Relationships with other nonlinear variables. Autonomic Neuroscience. 2013;177 2:271-4; doi: 10.1016/j.autneu.2013.05.004. 
47. Nguyen Phuc Thu T, Hernández AI, Costet N, Patural H, Pichot V, Carrault G, et al. Improving methodology in heart rate variability analysis for the premature infants: Impact of the time length. PLOE ONE. 2019;14 8:e0220692; doi: 10.1371/journal.pone.0220692.

48. Makowiec D, Wdowczyk J: Factor analysis of heart rate variability in healthy people. In: 11th Conference of the European Study Group on Cardiovascular Oscillations (ESGCO). 2020: 1-2.

49. Ciccone AB, Siedlik JA, Wecht JM, Deckert JA, Nguyen ND, Weir JP. Reminder: RMSSD and SD1 are identical heart rate variability metrics. Muscle \& Nerve. 2017;56 4:674-8; doi: 10.1002/mus.25573.

50. Kamen PW, Krum H, Tonkin AM. Poincaré plot of heart rate variability allows quantitative display of parasympathetic nervous activity in humans. Clinical science. 1996;91 2:201-8; doi: 10.1042/cs0910201.

51. Guzik P, Piskorski J, Krauze T, Schneider R, Wesseling K, Wykretowicz A, et al. Correlations between the Poincaré plot and conventional heart rate variability parameters assessed during paced breathing. The Journal of Physiological Sciences. 2007;57:63-71; doi: 10.2170/physiolsci.RP005506.

52. Huang TC, Lai TH, Taiwan Headache Society T. Medical treatment guidelines for preventive treatment of migraine. Acta Neurol Taiwan. 2017;26 1:33-53.

53. Allais G, De Lorenzo C, Quirico PE, Airola G, Tolardo G, Mana O, et al. Acupuncture in the prophylactic treatment of migraine without aura: A comparison with flunarizine. Headache. 2002;42 9:855-61; doi: 10.1046/j.15264610.2002.02203.x.

54. Karsan N, Palethorpe D, Rattanawong W, Marin J, Bhola R, Goadsby P. Flunarizine in migraine-related headache prevention: results from 200 patients treated in the UK. European Journal of Neurology. 2018;25 6:811-7; doi: 10.1111/ene.13621.

55. Luo N, Di W, Zhang A, Wang Y, Ding M, Qi W, et al. A randomized, one-year clinical trial comparing the efficacy of topiramate, flunarizine, and a combination of flunarizine and topiramate in migraine prophylaxis. Pain Medicine. 2012;13 1:80-6; doi: 10.1111/j.1526-4637.2011.01295.x.

56. Gidron Y, Deschepper R, De Couck M, Thayer JF, Velkeniers B. The vagus nerve can predict and possibly modulate non-communicable chronic diseases: introducing a neuroimmunological paradigm to public health. J Clin Med. 2018;7 371:1-11; doi: $10.3390 / \mathrm{jcm} 7100371$.

57. Choi KW, Jeon HJ. Heart rate variability for the prediction of treatment response in major depressive disorder. Front Psychiatry. 2020;11 607:1-7; doi: 10.3389/fpsyt.2020.00607.

58. Zhang P, Roberts T, Richards B, Haseler LJ. Utilizing heart rate variability to predict ICU patient outcome in traumatic brain injury. BMC Bioinformatics. 2020;21 481:1-11; doi: 10.1186/s12859-020-03814-w.

59. Ye J-J, Lee K-T, Lin J-S, Chuang C-C. Observing continuous change in heart rate variability and photoplethysmography-derived parameters during the process of pain production/relief with thermal stimuli. J Pain Res. 2017;10:527-33; doi: 10.2147/JPR.S129287.

60. Schurks M, Rist PM, Bigal ME, Buring JE, Lipton RB, Kurth T. Migraine and cardiovascular disease: Systematic review and meta-analysis. BMJ. 2009;339 3914:1-11; doi: 10.1136/bmj.b3914. 